\title{
Autophagy of intracellular microbes and mitochondria: two sides of the same coin? Vojo Deretic
}

Address: Department of Molecular Genetics and Microbiology, University of New Mexico Health Sciences Center, 915 Camino de Salud NE, Albuquerque, NM 87131, USA

Email: vderetic@salud.unm.edu

Fl000 Biology Reports 2010, 2:45 (doi:I0.34I0/B2-45)

The electronic version of this article is the complete one and can be found at: http://fl000.com/reports/biology/content/2/45

\begin{abstract}
Autophagy has become a biological paradigm of how eukaryotic cells, especially those that are long lived, maintain their vitality, control the quality of cytoplasmic organelles, and stay alive or die when growth factors are withdrawn and there is an energy or nutrient crisis. The role of autophagy has been extended to innate and adaptive immunity functions, which surpassed all initial expectations in terms of how immunity and autophagy are interconnected. Of particular interest at the moment is the growing appreciation of the similarity between how mitochondria and intracellular pathogens are handled by autophagy in its function of sanitizing the cytoplasm. An emerging framework from this may link the roots of cell defense against infection with cell longevity and programmed cell death.
\end{abstract}

\section{Introduction and context}

Autophagy is a fundamental biological process impacting a wide spectrum of human health and disease states [1], including cancer, neurodegeneration, myodegeneration, metabolic disorders (e.g., diabetes), aging, and innate and adaptive immunity [2]. The process of macroautophagy, or sensu stricto autophagy, can be viewed as a cytoplasmic homeostasis mechanism during which damaged or surplus intracellular organelles, supramacromolecular structures, large portions of the cytosol, and potentially toxic protein aggregates are sequestered into double membrane organelles termed autophagosomes [3]. Following the sequestration, the captured material is eliminated, when applicable, through maturation of autophagosomes into degradative organelles termed autolysosomes [3]. Very rapidly growing areas for autophagic investigations are the fields of immunity, infection, and inflammation. The field investigating the immunological roles of autophagy started with the appreciation that autophagy can clear intracellular microbes $[4,5]$ and has been significantly broadened since. Today, we know that autophagy contributes to a wide panel of innate and adaptive immunity processes, ranging from effector and regulatory functions in response to innate immunity receptor agonists [2], to development of naïve $\mathrm{T}$ cell repertoires [6], and major histocompatibility complex (MHC) II [7] and MHC I antigen presentation [8]. The immune systems intersect broadly with autophagy, posing the question of whether the two processes are connected in an evolutionarily ancient manner (thus explaining why autophagy is hardwired into so many immunological processes), or whether it is simply a convergent use of a versatile and successful process. The former model is more likely, a view that is supported by the latest wave of studies indicating a parallel between autophagy of mitochondria (a process termed mitophagy) and autophagy of intracellular microbes (a process known as xenophagy).

\section{Major recent advances}

When viewed as a crude biomass degradative process, autophagy can be a life-saving, cell-autonomous source of nutrients and energy produced by autodigestion of the cytosol under conditions where cells are limited for growth due to a lack of nutrients or growth factors. In its more sophisticated but also pro-survival manifestations, 
autophagy removes protein aggregates too large for proteasomal disposal, trims the amount and quality of intracellular compartments (e.g., endoplasmic reticulum) and disposes of damaged organelles, such as irreversibly depolarized or leaky mitochondria, that sporadically but relentlessly occur in all cells. The process of mitophagy, along with other aspects of quality control functions of autophagy, ensures survival and internal rejuvenation of long lived cells such as neurons, myocytes, macrophages, and so on. Furthermore, mitochondrial removal via autophagy takes place during development and cellular differentiation, as in the case of red blood cells [9] and T cell maturation [10]. Thus, mitophagy is key to both the longevity and proper differentiation and function of cells. The autophagy of stress-damaged mitochondria is based on the ubiquitination of proteins (e.g., voltage-dependent anion channel VDAC1) on depolarized mitochondria via the E3 ligase Parkin [11], which tags the mitochondria with poly-ubiquitinated chains. These are then recognized by the autophagic adapter protein p62 (SQSTM1) [12], which has a motif (WXXL) known as LIR (for LC3interacting region) that allows p62 to bind to LC3 (ATG8), a marquee protein for nascent autophagosomal membranes. The net result is that damaged mitochondria are 'reeled in' by the adapter p62 into the autophagic organelles for clearance [11] (Figure 1A).

Intriguingly, a similar mechanism has been nearly simultaneously described for autophagic removal of a number of intracellular bacteria (Figure 1B) and potentially other microbes. Sindbis virus capsid is recognized by p62 and targeted to autophagosomes protecting infected neurons from virus-induced pathology [13]. Polyubiquitin-decorated Listeria (rendered defenseless by the loss of ActA) is recognized by p 62 and delivered to autophagosomes for degradation [14]. The perforated phagosomes during escape of Shigella into the cytosol are also captured for autophagy by polyubiquitin-p62-LC3 bridges [15]. Interestingly, when autophagic clearance of damaged Shigella phagosomal remnants was obstructed, TRAF6 (known to directly interact with p62), led to inflammatory signaling and cell death [15], suggesting a potential scenario where if the pathogen or signals associated with its presence in the cytosol are not eliminated by autophagy, a second stage response kicks in leading to elimination of the chronically infected cell, thus limiting infection spread by cell death. The adapter p62 is not the only participant in these processes. Whereas Salmonella can be autophagically eliminated in a similar process using p62 [16], another LC3-interacting adapter, nuclear dot protein 52 (NDP52), is a key player in removing cytosolic Salmonella [17]. Apart from Salmonella, NDP52 plays a role in autophagy of cytosolic Streptococcus pyogenes [17]. In some cases, as with Mycobacterium tuberculosis that resides inside the phagosomes in infected macrophages, it is not the microbe that is captured by $\mathrm{p} 62$ and delivered to autophagosomes, but rather p62 plays a role in antimicrobial action of autophagy in a different, mirror image process [18]. In this case, p62 collects cytoplasmic proteins, such as ubiquitin or ribosomal precursor proteins, and delivers them to autophagosomes

Figure I. Conservation of the process of autophagic removal of mitochondria and intracellular bacteria

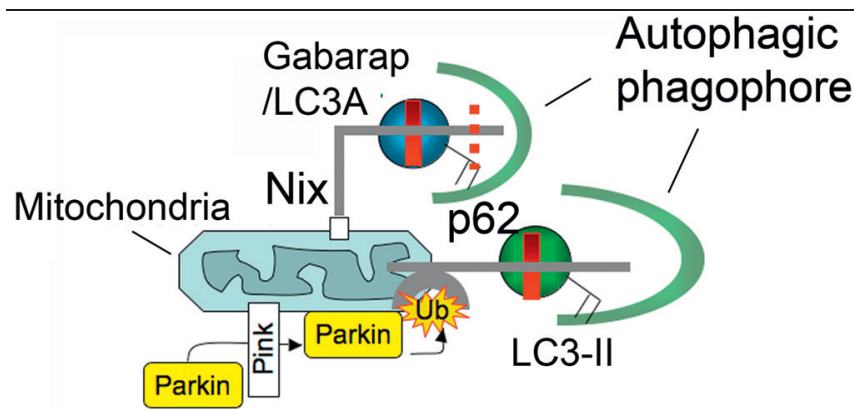

A

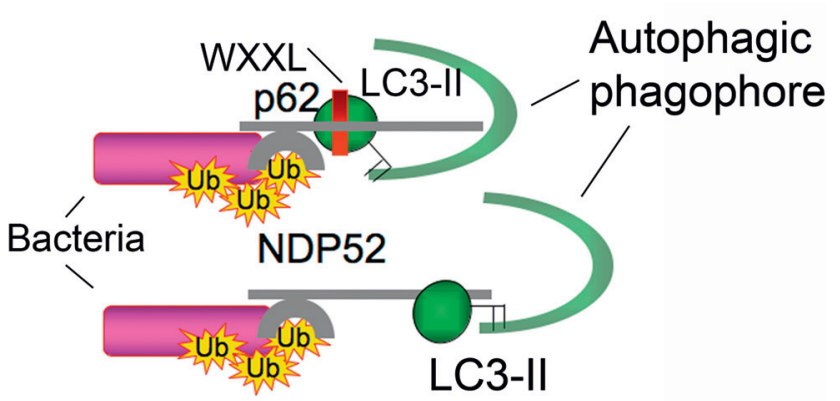

B

The process involves an autophagic adapter (e.g., p62, NDP52 [nuclear dot protein 52], or Nix), and a mammalian Atg8 paralog (LC3, Gabarap) that binds to the adapter via a WXXL motif, thus bringing the targeted cargo (mitochondria, bacteria) into the nascent autophagic organelle, termed the phagophore; the phagophore grows, wraps around the target, and eventually closes, leading to sequestration and elimination. (A) Mitochondria in mammalian cells are removed by autophagy via the Nix adapter (also known as BNIP3L) during developmental elimination of mitochondria, or via ubiquitination of VDACI (voltage-dependent anion channel I; see text) recognized by the adapter p62 for removal of stressed (e.g., depolarized or damaged) mitochondria. (B) Intracellular bacteria exposed to the cytosol are decorated by ubiquitin (Ub) and recognized by autophagic adapters (p62 or NDP52) for sequestration into autophagosomes and elimination. 
where they are digested into smaller peptides within the autolysosomes. This autolysosomal peptide cargo is then delivered to mycobacterial phagosomes. It turns out that ribosomal or ubiquitin fragments proteolytically generated in autophagic organelles from otherwise innocuous precursor cytoplasmic proteins possess antimicrobial properties and help kill intracellular M. tuberculosis [18].

\section{Future directions}

The similarity of the molecular processes involved in autophagic targeting of mitochondria and microbes suggests a potentially common evolutionary root of mitophagy and xenophagy. Since mitochondria evolved from a Rickettsia-like $\alpha$-protobacterium, it is possible that the processes we recognize today as mitophagy or xenophagy (and perhaps autophagy altogether) may have diverged from a common primordial system (proto-autophagy) in early eukaryotic cells that had to deal with invasion by intracellular microbes. If this model is correct, understanding the nature of interactions between cells and mitochondria may help understand immunological roles of autophagy. Likewise, cell survival and programmed cell death processes (dependent on mitochondria), of high significance for many normal or pathological states such as neurodegeneration, cancer, and aging, may conceptually and mechanistically benefit from understanding the common proto-autophagy roots of mitophagy and autophagic elimination of microbes.

\section{Abbreviations}

ATG8, autophagy-related protein 8; MHC, major histocompatibility complex; NDP52, nuclear dot protein 52; SQSTM1, sequestosome 1; TRAF6, tumor necrosis factor receptor-associated factor 6 .

\section{Competing interests}

The author declares that he has no competing interests.

\section{Acknowledgments}

This work was supported by grants RC1AI086845, AI069345, and AI42999 from the National Institutes of Health, 107160-44-RGRL from the American Foundation for AIDS Research (amfAR), a Bill \& Melinda Gates Grand Challenge Explorations grant, and a grant from the Crohn's and Colitis Foundation of America.

\section{References}

I. Mizushima N, Levine B, Cuervo AM, Klionsky DJ: Autophagy fights disease through cellular self-digestion. Nature 2008, 45 I: 1069-75.

2. Deretic V, Levine B: Autophagy, immunity, and microbial adaptations. Cell Host Microbe 2009, 5:527-49.

3. Mizushima N, Yoshimori T, Levine B: Methods in mammalian autophagy research. Cell 2010, 140:313-26.
4. Gutierrez MG, Master SS, Singh SB, Taylor GA, Colombo MI, Deretic V: Autophagy is a defense mechanism inhibiting BCG and Mycobacterium tuberculosis survival in infected macrophages. Cell 2004, I I 9:753-66.

FI000 Factor 6.5 Must Read

Evaluated by Suresh Subramani 25 Nov 2004, Gerald Pier 2I Dec 2004, Michael Gold 23 Dec 2004

5. Nakagawa I, Amano A, Mizushima N, Yamamoto A, Yamaguchi H, Kamimoto T, Nara A, Funao J, Nakata M, Tsuda K, Hamada S, Yoshimori T: Autophagy defends cells against invading group A Streptococcus. Science 2004, 306: 1037-40.

FI000 Factor 4.8 Must Read

Evaluated by Daniel Klionsky 09 Nov 2004, Suresh Subramani 25 Nov 2004

6. Nedjic J, Aichinger M, Emmerich J, Mizushima N, Klein L: Autophagy in thymic epithelium shapes the T-cell repertoire and is essential for tolerance. Nature 2008, 455:396-400.

FI000 Factor 6.8 Must Read

Evaluated by Vojo Deretic 27 Aug 2008, Edward Collins 03 Oct 2008, Thomas Huenig 09 Oct 2008, Colin Watts 17 Oct 2008

7. Jagannath $C$, Lindsey $D R$, Dhandayuthapani $S, X u$, Hunter RL Jr, Eissa NT: Autophagy enhances the efficacy of BCG vaccine by increasing peptide presentation in mouse dendritic cells. Nat Med 2009, 15:267-76.

FI000 Factor 8.I Exceptional

Evaluated by Vojo Deretic 04 Mar 2009, Thomas W von Geldern 24 Mar 2009, George Yap 09 Apr 2009

8. English L, Chemali M, Duron J, Rondeau C, Laplante A, Gingras D, Alexander D, Leib D, Norbury C, Lippé R, Desjardins M: Autophagy enhances the presentation of endogenous viral antigens on MHC class I molecules during HSV-I infection. Nat Immunol 2009, 10:480-7.

FI000 Factor 6.0 Must Read

Evaluated by George Yap 09 Apr 2009

9. Sandoval H, Thiagarajan P, Dasgupta SK, Schumacher A, Prchal JT, Chen M, Wang J: Essential role for $\mathbf{N i x}$ in autophagic maturation of erythroid cells. Nature 2008, 454:232-5.

FI000 Factor 8.I Exceptional

Evaluated by Richard Youle 13 May 2008, Christoph Borner 27 May 2008, Daniel Klionsky 2I Aug 2008

10. Pua HH, Guo J, Komatsu M, He YW: Autophagy is essential for mitochondrial clearance in mature $\mathrm{T}$ lymphocytes. J Immunol 2009, 182:4046-55.

II. Geisler S, Holmstrom KM, Skujat D, Fiesel FC, Rothfuss OC, Kahle PJ, Springer W: PINKI/Parkin-mediated mitophagy is dependent on VDACI and p62/SQSTMI. Nat Cell Biol 2010, I 2:|| |9-3|.

FI000 Factor 6.0 Must Read

Evaluated by Ivan Dikic 16 Feb 2010

12. Kirkin V, Lamark T, Sou YS, Bjørkøy G, Nunn JL, Bruun JA, Shvets E, McEwan DG, Clausen TH, Wild P, Bilusic I, Theurillat JP, Øvervatn A, Ishii T, Elazar Z, Komatsu M, Dikic I, Johansen T: A role for NBRI in autophagosomal degradation of ubiquitinated substrates. Mol Cell 2009, 33:505-16.

FI000 Factor 3.0 Recommended

Evaluated by Daniel Klionsky 0I Jun 2009

13. Orvedahl A, Macpherson S, Sumpter R Jr, Talloczy Z, Zou Z, Levine B: Autophagy protects against Sindbis virus infection of the central nervous system. Cell Host Microbe 2010, 7:1 15-27.

14. Yoshikawa $Y$, Ogawa M, Hain T, Yoshida M, Fukumatsu M, Kim M, Mimuro H, Nakagawa I, Yanagawa T, Ishii T, Kakizuka A, Sztul E, Chakraborty T, Sasakawa C: Listeria monocytogenes 
ActA-mediated escape from autophagic recognition. Nat Cell Biol 2009, II:I233-40.

Evaluated by 6.0 Must Read

Evaluated by Pascale Cossart 28 Oct 2009

15. Dupont N, Lacas-Gervais S, Bertout J, Paz I, Freche B, Van Nhieu GT, van der Goot FG, Sansonetti PJ, Lafont F: Shigella phagocytic vacuolar membrane remnants participate in the cellular response to pathogen invasion and are regulated by autophagy. Cell Host Microbe 2009, 6:137-49.

FI000 Factor 6.0 Must Read

Evaluated by Vojo Deretic 20 Nov 2009

16. Zheng YT, Shahnazari S, Brech A, Lamark T, Johansen T, Brumell JH: The adaptor protein p62/SQSTMI targets invading bacteria to the autophagy pathway. J Immunol 2009, 183:5909-16.
17. Thurston TL, Ryzhakov G, Bloor S, von Muhlinen N, Randow F: The TBKI adaptor and autophagy receptor NDP52 restricts the proliferation of ubiquitin-coated bacteria. Nat Immunol 2009, 10:|2|5-2|.

FI000 Factor 3.0 Recommended

Evaluated by Daniel Klionsky 13 Nov 2009

18. Ponpuak M, Davis AS, Roberts EA, Delgado MA, Dinkins C, Zhao Z, Virgin HW 4th, Kyei GB, Johansen T, Vergne I, Deretic V: Delivery of cytosolic components by autophagic adapter portein p62 endows autophagosomes with unique antimicrobial properties. Immunity 2010, 32:329-4I.

F1000 Factor 6.0 Must Read

Evaluated by Michel Desjardins II Mar 2010 